\section{Säuglingsnahrung nach dem Vorbild der Muttermilch}

$M$ uttermilch ist die optimale Nahrung für Säuglinge. Denn mit ihren präbiotischen Inhaltsstoffen und probiotischen Kulturen sorgt sie für eine gesunde Darmflora, die eine Schlüsselrolle bei der Entwicklung der Darmfunktion und des Immunsystems spielt. Aufbauend auf den Erkenntnissen der Muttermilch-Forschung hat das Unternehmen Hipp jetzt mit Hipp Combiotik $^{\circledR}$ eine neue Generation der Milchnahrung entwickelt, die Inhaltsstoffe nach dem Vorbild der Muttermilch enthält. Dabei ist es erstmals gelungen, die Kombination aus dem Probiotikum Lactobacillus fermentum hereditum ${ }^{\circledR}$, einer aus der Muttermilch isolierten probiotischen Kultur, und dem Präbiotikum Galacto-Oligosaccharid (GOS) in einer Säuglingsnahrung zu vereinen.

Präbiotika sind Substanzen, in der Regel Kohlenhydrate, die vom menschlichen Organismus weder verdaut noch absorbiert werden. Sie gelangen in tiefere Darmabschnitte, werden von der dort ansässigen Mikroflora fermentiert und stimulieren so selektiv deren Vermehrung. Zu dieser Mikroflora zählen nicht-pathogene Lactobacillen und Bifi-

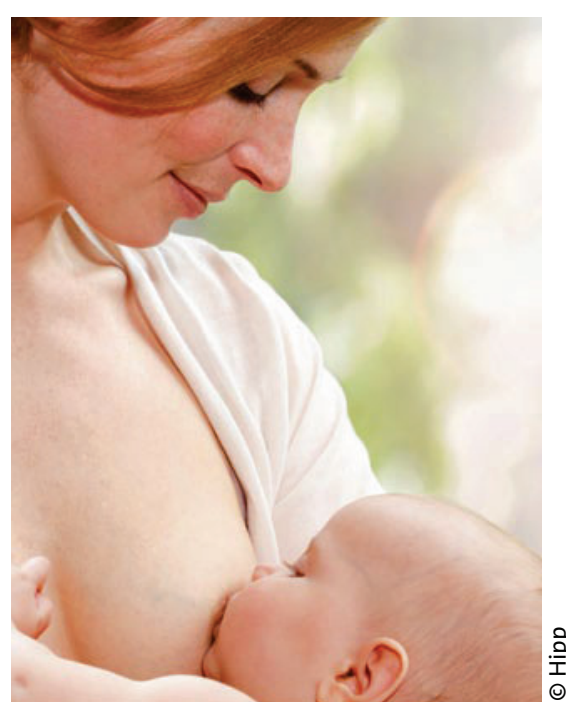

Muttermilch ist das Vorbild für eine neue Generation der Säuglingsnahrung, die das Probiotikum Lactobacillus fermentum hereditum $^{\circledR}$ mit dem dem Präbiotikum Galacto-Oligosaccharid kombiniert. duskeime, die bei erhöhtem Bedarf zusätzlich über probiotische Zubereitungen aufgenommen werden können.

Zwei doppelblinde randomisierte klinische Studien bestätigten die Sicherheit, gute Verträglichkeit und Effektivität der Kombination von L. fermentum hereditum $^{\circledR}$ und GOS. Unter der Leitung von Dr. Eduardo López-Huertas vom Spanish Council for Scientific Research, Granada, standen bei der ersten Studie Folgenahrungen für Kinder zwischen sechs und zwölf Monaten im Zentrum des Interesses. Kinder, die diese Kombination erhalten hatten, gediehen altersgemäß, erlitten signifikant weniger Durchfälle und entwickelten weniger Atemwegserkrankungen als eine Kontrollgruppe, die Folgenahrung ausschließlich mit GOS erhalten hatte. Gegenstand der zweiten Studie waren Anfangsnahrungen für Neugeborene. Auch bei dieser Gruppe war die Inzidenz von Durchfällen bei Ernährung mit der Kombination aus Pro- und Präbiotikum signifikant niedriger. Wachstum und Gedeihen waren altersgemäß und ohne Unterschied zur Kontrollgruppe.

Die neue Nahrung gibt es auch für Kinder mit Allergierisiko: Hier empfiehlt sich Hipp HA Combiotik ${ }^{\circledR}$ als hypoallergene Anfangs- und Folgenahrung. Das darin enthaltene Kuhmilcheiweiß wurde durch Hydrolyse in kleinere Einheiten gespalten. Nach einer prospektiven Beobachtungsstudie (Klinische Pädiatrie 2009; 221: 78-82) war das Risiko, eine atopische Dermatitis in den zwei ersten Lebensjahren zu entwickeln, bei initial gestillten oder vorwiegend mit einem Eiweißhydrolysat ernährten Kindern vergleichbar gering. Am Ende des ersten Lebensjahres lag kein signifikanter Unterschied im SCORAD beider Gruppen vor. Nach dem sechsten Monat können Eltern die hypoallergene Ernährung mit Hipp HA 2 Combiotik $^{\circledR}$ und ab dem zehnten Monat mit Hipp HA 3 Combiotik $^{\circledR}$ fortsetzen.

red

Nach Informationen von Hipp, Pfaffenhofen

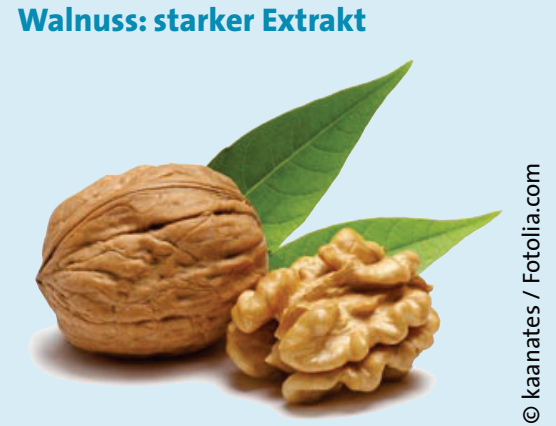

Im Rahmen der kontinuierlichen Verbesserung der Nahrungsmittelallergene der ImmunoCAP ${ }^{\circledR}$-Reihe hat deren Hersteller Phadia den Extraktionsprozess für das Walnussallergen $\mathrm{f} 256$ optimiert. Wichtige Komponenten wie das Speicherprotein Jug $r 1$ - ein 2S-Albumin und Hauptallergen der Pflanze - können nun vermehrt extrahiert und gekoppelt werden. In einer internen Studie zeigte sich schon vorab, dass die Nachweisempfindlichkeit damit deutlich erhöht werden kann. Das verbesserte Allergen ist seit Anfang 2011 in einer Packungsgröße von 16 Testkits pro Röhrchen verfügbar.

red

Nach Informationen von Phadia, Freiburg

\section{Beruhigende Nasendusche}

Da Patienten mit allergischer Rhinitis das Atmen nicht einfach einstellen können, ist das Eindringen der Pollen in die Nase kaum zu verhindern. Bei dem Versuch, die Fremdkörper aus der Nase wieder zu entfernen, hilft die Emser ${ }^{\Theta_{-}}$ Nasendusche, die mit einer isotonischen Nasenspülsalz-Lösung gefüllt wird. Nach dem Ausspülen der Allergieauslöser beruhigt sich die gereizte Nasenschleimhaut und die Symptome werden gelindert. Bei der Anwendung läuft die Spüllösung zu einem Nasenloch hinein, zum anderen wieder hinaus. So werden neben den Allergenen auch Krankheitskeime und Verunreinigungen entfernt. Wichtig ist die Wahl des richtigen Mineralsalzes zur Herstellung der Spüllösung. Hierzu eignet sich z. B. Emser ${ }^{\circledR}$-Nasenspülsalz, das in Packungsgrößen von 20, 50 oder 100 einfach zu handhabenden Portionsbeuteln in der Apotheke erhältlich ist. Sein physiologischer Anteil an Hydrogencarbonat unterstützt die Schleimlösung. Weitere enthaltene Mineralstoffe pflegen die Nasenschleimhaut und tragen dazu bei, dass die Nase über Stunden frei bleibt.

NachInformationen von Siemens \& Co, Bad Ems 